\title{
Annular Dark Field Imaging of Catalyst Nanoparticles in Single Shot Dynamic Transmission Electron Microscopy
}

\author{
D.J. Masiel*, B. Reed**, T. LaGrange**, N.D. Browning** \\ *University of California, Davis, 1 Shields Avenue, Davis, CA 95616, USA \\ **Lawrence Livermore National Laboratory, 7000 East Avenue, Livermore, CA 94550, USA
}

It is currently possible to achieve spatial resolutions of up to $8 \mathrm{~nm}$ with $15 \mathrm{~ns}$ electron pulses in Dynamic Transmission Electron Microscopy (DTEM) using high contrast samples such as gold and carbon multilayer films [1]. DTEM has the potential to offer unprecedented insight into the dynamics of catalytic processes, however low signal to background levels and stochastic blur currently limit the achievable single shot spatial resolution in such systems [2]. To approach the 8nm resolution obtained in the gold and carbon multilayer system it would be advantageous to achieve comparable contrast levels for imaging catalyst nanoparticles on low Z substrates [3]. Annular dark field imaging using an annular aperture in the back focal plane of the objective lens is a viable approach to improving the contrast in pulsed electron imaging of catalyst systems [4].

Annular apertures with an outer diameter of $264 \mu \mathrm{m}$ and an inner diameter of $137 \mu \mathrm{m}$ were fabricated from $12.5 \mu \mathrm{m}$ thick tantalum foils. In the DTEM at Lawrence Livermore National Laboratory this geometry corresponds to an angle of 20mradians at the edge of the central disc and an angle of 40 mradians at the edge of the outer aperture hole. A $1 \mu \mathrm{m}$ layer of aluminum was deposited on the foils to serve as a hard mask. The aluminum hard mask was then patterned by photolithography. The exposed tantalum was subsequently removed using reactive ion etching in sulfur hexafluoride plasma to produce an annular dark field aperture with three support arms.

$\mathrm{Co}_{3} \mathrm{O}_{4}$ nanoparticles are used to catalyze the growth of silica nanocoils [5]. These particles show relatively low contrast on a carbon support in single shot pulsed imaging, and as such are an ideal catalyst system for testing the efficacy of annular dark field DTEM. The results of initial tests using this system clearly show that annular dark field DTEM vastly enhances both the signal to background ratio and contrast of single shot pulsed electron images.

References:

[1] King, W., Armstrong, M., Bostanjoglo, O., Reed, B. Science of Microscopy, Springer, New York (2007) 406.

[2] M.R. Armstrong et al., Ultramicroscopy 107 (2007) 365.

[3] N.L. Okamoto et al., J. of Phy. Chem. C 112 (2008) 1759.

[4] S. Bals et al., Sol. State Comm. 130 (2004) 675.

[5]Y. Qu et al., J. of Colloid and Interface Science. 321 (2008) 251.

[6] This work was supported by DOE/NNSA award DE-PS52-05NA (N. Browning [PI], W.A.

Schroeder, J. Spence) and was performed in part under the auspices of the U.S. Department of Energy Office of Science, Office of Basic Energy Sciences, Division of Materials Sciences and Engineering by Lawrence Livermore National Laboratory under Contract DE-AC52-07NA27344 


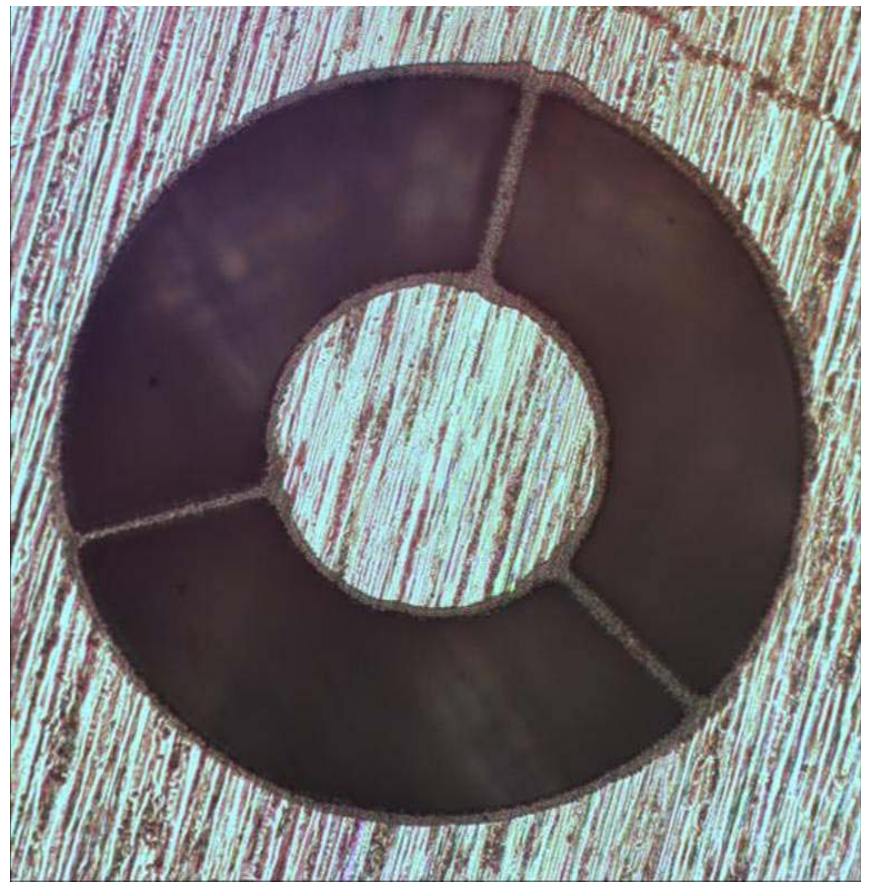

Fig. 1. Optical microscope image of a plasma etched, tantalum annular objective aperture

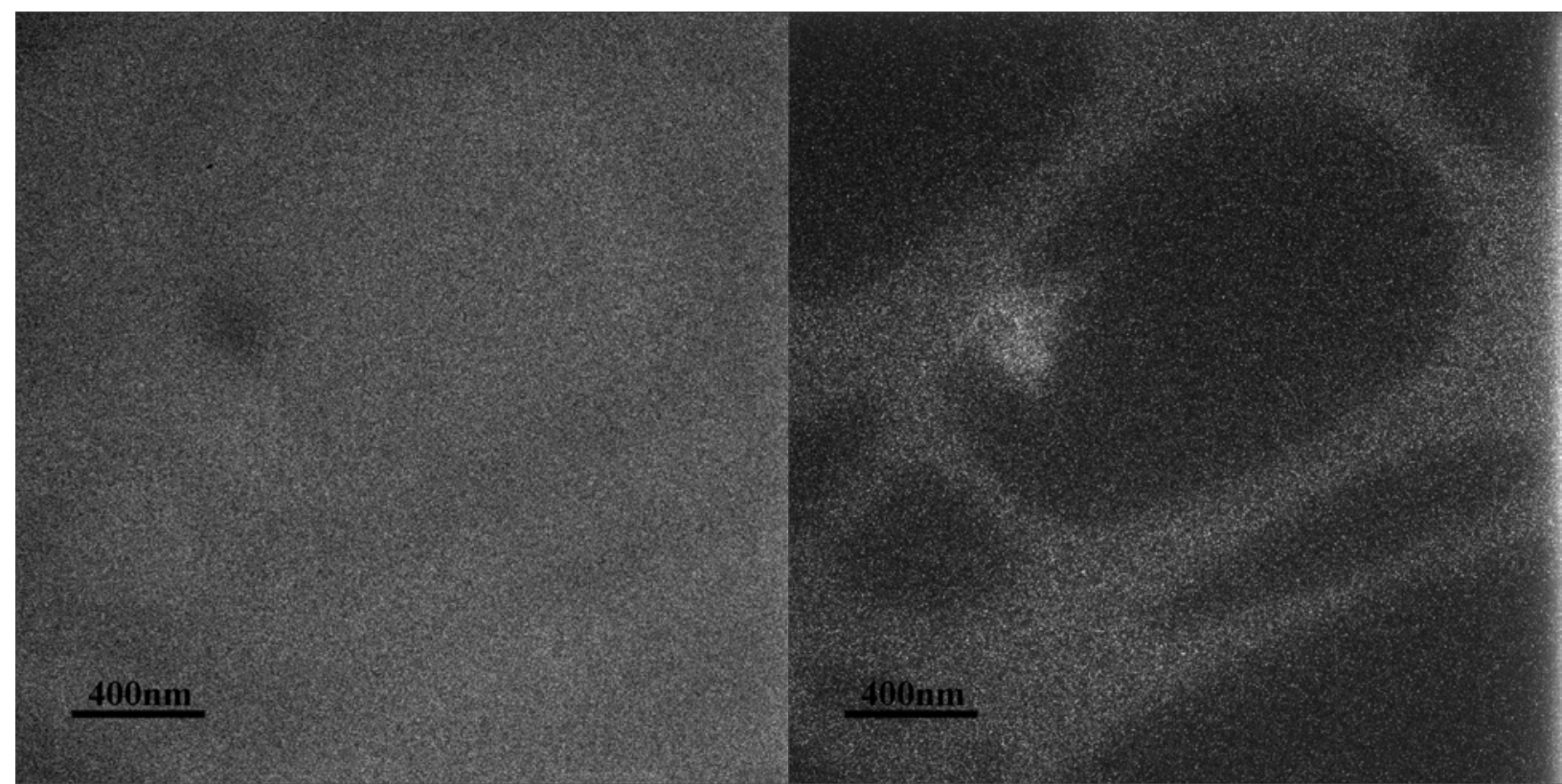

Fig. 2. Bright field (left) and annular dark field (right) images of a cluster $\mathrm{Co}_{3} \mathrm{O}_{4}$ nanoparticles on a lacey carbon grid with formvar backing. Each image was taken using a single $15 \mathrm{~ns}$ electron pulse. 